\title{
POTENCIAL QUANTITATIVO E QUALITATIVO DE GENÓTIPOS BATATA-DOCE
}

\author{
Quantitative and qualitative potential of sweet potato genotypes
}

\author{
Valter Carvalho de Andrade Júnior ${ }^{1}$, Ahmed Youssef A M Elsayed ${ }^{2}$, Alcinei Mistico Azevedo ${ }^{3 *}$, Edson Aparecido dos Santos ${ }^{2}$, \\ Marcos Aurélio M Ferreira ${ }^{4}$ \\ ${ }^{1}$ Docente; Departamento de agricultura; Universidade Federal de Lavras. Campus da UFLA, CEP 37200-000, Lavras, MG, Brasil. \\ valter.andrade@dag.ufla.br \\ ${ }_{2}^{2}$ Doutor; Departamento de agronomia; Universidade Federal dos Vales do Jequitinhonha e Mucuri. Campus JK, CEP 39100-000, \\ Diamantina, MG. ahmedtohy@yahoo.com; edsonapsant@yahoo.com.br \\ ${ }^{3}$ Docente; Instituto de Ciências Agrárias; Universidade Federal de Minas Gerais. Campus Montes Claros, CEP 39404-547, Montes \\ Claros, MG. alcineimistico@hotmail.com (Autor para correspondência) \\ ${ }^{4}$ Mestrando; Departamento de agronomia; Universidade Federal dos Vales do Jequitinhonha e Mucuri. Campus JK, CEP 39100-000, \\ Diamantina, MG.marcaumife2010@hotmail.com
}

Artigo enviado em 13/01/2017, aceito em 31/07/2017 e publicado em 10/04/2018.

Resumo: Não há na literatura trabalhos que avaliem descritores importantes em batata-doce individualmente em ramas, raízes e em toda a planta (raízes + ramas). Esta informação detalhada é importante, pois permite a seleção dos melhores genótipos considerando a planta (raízes + ramas), tendo em vista as diferentes formas de utilização: consumo humano, animal, indústria e produção de etanol. Portanto, objetivou-se avaliar o potencial produtivo de matéria fresca, matéria seca, amido e proteína bruta em raízes, ramas e planta (ramas + raízes) de batata-doce, a fim de se identificar os genótipos superiores. Para isso, foram avaliados 40 acessos de batata-doce do banco de germoplasma da UFVJM. A produção total da batata-doce (raízes + ramas) foi alta, o que aliado a sua rusticidade e múltiplos fins realça seu grande potencial e importância. Houve grande variabilidade genética entre os acessos. Os acessos Tomba Carro 1, UFVJM (01, 28 e 40) são os mais recomendados devido as maiores produtividades de raízes, ramas e proteína bruta, sendo adequados à alimentação humana e animal. Os acessos Palmas e UFVJM (01, 04, 05, 07 e 53) podem ter maior potencial para a produção de etanol devido à maior produtividade de amido.

Palavras-Chave - Ipomoea batatas (L.) Lam., germoplasma; melhoramento genético, alimentação humana, alimentação animal.

\begin{abstract}
There are no studies in the literature that evaluate important traits in sweet potatoes individually in branches, roots and the whole plant (roots + branches). This detailed information is important because it allows the selection of the best genotypes considering the plant (roots + branches), taking into account the different uses: food, feed, industry and ethanol production. Therefore, this study aimed to evaluate the production potential of fresh weight, dry matter, crude protein and starch in roots, branches and plant (branches + roots) of sweet potatoes, in order to identify superior genotypes. For this, we evaluated 40 sweet potato accessions of germplasm bank of UFVJM. The total production of sweet potatoes (roots + hamas) was high, which combined with its rusticity and multipurpose highlights its great potential and importance. There was great genetic variability among accessions. The Tomba Carro 1, UFVJM (01, 28 and 40) are the most recommended because of the higher yields of roots, branches and crude protein, being suitable for human and animal consumption. The Palma and UFVJM (01, 04, 0507 and 53) accesses may have greater potential for ethanol production due to the high starch yield.
\end{abstract}

Keywords - Ipomoea batatas (L.) Lam., germplasm, breeding, food, animal feed. 


\section{INTRODUÇÃO}

A batata-doce (Ipomoea batatas (L.) Lam.) é uma hortaliça tuberosa de grande importância econômica e social. Aproximadamente $85 \%$ da produção mundial é produzida no continente asiático, com destaque para a China, que produz aproximadamente 79 milhões de toneladas de raízes anualmente, com produtividade média de 22,44 $\mathrm{t} \mathrm{ha}^{-1}$ (FAO 2013). No Brasil, a área plantada é de 39.000 ha, com produção de 479 mil toneladas e rendimento médio de 12,28 t ha ${ }^{-1}$ (FAO 2013). Esta produtividade é considerada baixa, o que pode ser consequência do desconhecimento de práticas de manejo adequadas, da utilização de materiais obsoletos, do baixo nível tecnológico empregado, entre outras (GONÇALVES NETO et al., 2011; AZEVEDO et al., 2014).

Embora a produtividade brasileira de batatadoce seja baixa, altas produtividades de raízes não são raras na literatura nacional, como $45,4 \mathrm{t} \mathrm{ha}^{-1}$ (ANDRADE JÚNIOR et al., 2009), 51,0 t ha-1 (AZEVEDO et al., 2014) e 98,0 $\mathrm{t} \mathrm{ha}^{-1}$ (GONÇALVES NETO et al., 2011). Segundo Andrade Júnior et al. (2009) e Azevedo et al. (2014), produtividades superiores a $30 \mathrm{t} \mathrm{ha}^{-1}$ podem ser facilmente obtidas com o plantio de genótipos com bom potencial produtivo. Altas produtividades de matéria fresca de ramas também são encontradas como 15,7 $\mathrm{t}$ ha-1 (ANDRADE JÚNIOR et al., 2012), 23,9 $\mathrm{t} \mathrm{ha}^{-1}$ (FIGUEIREDO et al., 2012) e 64,5 $\mathrm{t} \mathrm{ha}^{-1}$ (VIANA et al., 2011). Além das raízes de batata-doce serem geralmente destinadas à alimentação humana, seu uso também pode ser direcionado à produção de etanol ou à alimentação animal junto com as ramas (GONÇALVES NETO et al., 2011). Logo, a cultura apresenta múltiplos fins (AZEVEDO et al., 2014), e consequentemente, possui alta importância para a segurança alimentar.

\section{MATERIAL E MÉTODOS}

O experimento foi instalado no Setor de Olericultura, localizado no campus JK da Universidade Federal dos Vales do Jequitinhonha e Mucuri (UFVJM), em Diamantina/MG. Foram avaliados 40 acessos de batata-doce pertencentes ao banco de germoplasma da UFVJM, sendo eles a Batata Mandioca, Brazlândia Branca, Brazlândia Rosada, Cambraia, Cariru Vermelha, Espanhola, Licuri, Palmas, Tomba Carro1 e UFVJM (01, 02, 04, 05, 06, $07,12,14,18,20,21,23,24,25,28,38,39,40,41,44$, $46,47,48,50,51,52,53,55,56,57$ e 58$)$.
No Brasil é crescente o número de experimentos avaliando genótipos de batata-doce quanto à resistência a pragas e doenças (MARCHESE et al., 2010); melhor qualidade nutricional (ANDRADE JÚNIOR et al., 2012; AZEVEDO et al., 2014); maior densidade de raízes (CARDOSO et al., 2012); maior aptidão para produção de etanol (GONÇALVES NETO et al., 2011); maior produção de raízes para alimentação humana (AZEVEDO et al., 2014) e maior produção de ramas para alimentação animal (VIANA et al., 2011; ANDRADE JÚNIOR et al., 2012). Porém, não há trabalhos na literatura avaliando o potencial da cultura da batata-doce considerando isoladamente a produtividade de matéria fresca, de matéria seca, de proteína bruta e amido nas raízes, ramas e na planta (ramas + raízes). Esta informação detalhada é importante, pois permite a seleção dos melhores genótipos de forma completa, tendo em vista as diferentes formas de utilização da cultura: consumo humano, animal, indústria e produção de etanol.

O conhecimento da produtividade de matéria fresca e seca de raízes e ramas de batata-doce é o principal descritor para a seleção de genótipos superiores agronomicamente. Já a proteína bruta possibilita inferir sobre a boa digestibilidade na alimentação animal, tanto na forma fresca ou como silagem (MONTEIRO, 2007). Enquanto isso, o amido é o principal carboidrato de reserva produzido pelas plantas e consumido pelo homem, tendo ampla possibilidade de utilização diretamente na dieta humana, na indústria alimentícia (CORDENUNSI et al., 2005) e na produção de etanol (LÁZARI et al., 2014). Desta forma, objetivou-se avaliar o potencial produtivo de matéria fresca, matéria seca, amido e proteína bruta em raízes, ramas e na planta (ramas + raízes) de batata-doce, a fim de se identificar genótipos superiores.

Para o plantio foram selecionadas ramas sadias contendo entre seis e oito gemas. Estas ramas foram plantadas em bandejas de isopor de 72 células, preenchidas com substrato comercial no dia 13 de novembro de 2012. As mudas foram mantidas em casa de vegetação por cinco semanas e irrigadas por microaspersão, sendo posteriormente transplantadas para o campo. O experimento foi implantado no delineamento em blocos casualizados com espaçamento de 0,30 metros entre plantas e 1 metro entre linhas, sendo cada parcela composta por oito plantas. Foram avaliados 40 acessos de batata-doce com quatro repetições. Aos 30 dias antes do transplantio, foi feita uma aração e duas gradagens, seguidas de sulcamento para formação das leiras. O 
solo é do tipo Neossolo quartizarênico órtico típico. Os resultados da análise química antes da implantação do experimento foram: $\mathrm{pH}$ (água) - 5,8; P e K (mg dm$\left.{ }^{3}\right)$ - 1,1 e 6,3 respectivamente; $\mathrm{SB}, \mathrm{Ca}, \mathrm{Mg}, \mathrm{Al}$ e $\mathrm{H}+\mathrm{Al}$ $\left(\right.$ cmolc $\left.\mathrm{dm}^{-3}\right)-1,1 ; 0,6 ; 0,5 ; 0,2$ e 2,4 respectivamente; $\mathrm{V}(\%)-32 ; \mathrm{MO}$, areia, argila e silte $\left(\mathrm{dag} \mathrm{kg}^{-1}\right)-0,86,6$ e 8 , respectivamente. As adubações de plantio e cobertura foram realizadas de acordo com o recomendado para a cultura.

Aos 180 dias após o transplantio foram avaliadas as produtividades total e comercial de raízes e a produtividade de massa fresca das ramas. Foram consideradas como comercializáveis raízes entre 0,1 e 0,8 quilos sem rachaduras, deformações, esverdeamentos, danos por insetos ou presença de veias. Tais estimativas foram obtidas considerando o somatório da produção de cada parcela. Amostras de raízes e ramas de 200 gramas foram retiradas para avaliação de seus respectivos teores de matéria seca, após secagem em estufa com circulação de ar, em temperatura de $65{ }^{\circ} \mathrm{C}$ até peso constante (aproximadamente 72 horas). As produtividades de massa seca de ramas e raízes também foram determinadas, sendo os resultados expressos em $\mathrm{t} \mathrm{ha} \mathrm{a}^{-1}$. A partir das amostras de matéria seca, foram determinados os teores de proteína bruta nas raízes e ramas. Já o teor de amido das raízes foi obtido recorrendo-se à metodologia apresentada por Mccready et al. (1950). Após a obtenção do teor (\%) de amido e proteína, os valores obtidos foram convertidos para t ha ${ }^{-1}$.

Os dados foram submetidos à análise de variância e as médias dos tratamentos foram comparadas utilizando-se o teste de agrupamento de Scott-Knott em nível de 5\% de significância.

\section{RESULTADOS E DISCUSSÃO}

Foi observada significância para o efeito dos tratamentos em nível de 5\% para todas as características avaliadas, exceto para o teor de amido nas raízes. Grandes desvios entre os valores mínimos e máximos foram encontrados para os acessos de batata-doce avaliados em todas as características, confirmando a presença de variabilidade. As características que apresentaram maior coeficiente de variação foram a produtividade de amido nas raízes, produtividade de matéria fresca de ramas e produtividade de proteína bruta nas ramas (Tabela 1). Azevedo et al. (2015) também encontraram alta estimativa do coeficiente de variação para a produtividade de matéria fresca de ramas $(61,61 \%)$.

Altas estimativas de correlação foram encontradas entre as características produtividade total de raízes, produtividade comercial de raízes, produtividade de matéria seca das raízes, produtividade de proteína bruta nas raízes e produtividade de matéria seca total (Tabela 1). Estas altas estimativas de correlações são esperadas, pois todas se relacionam diretamente ou indiretamente com a produção de raízes. Por motivo similar, também foi encontrada alta estimativa de correlação entre produtividade de matéria fresca de ramas e produtividade de matéria seca total.

Os acessos Batata Mandioca, Cariru Vermelha, Licuri, Tomba Carro1 e UFVJM (01, 06, 21, $23,28,40$ e 56) apresentaram os maiores valores para produtividade total de raízes, com rendimento de até 47,1 $\mathrm{t} \mathrm{ha}^{-1}$ (Tabela 2). Além destes genótipos, a cultivar Brazlândia Rosada também se destacou entre os genótipos com maior produtividade comercial de raízes. Estes acessos apresentaram produtividade média de raízes comerciais de 27,46 $\mathrm{t} \mathrm{ha}^{-1}$, muito superior à média nacional $\left(12,28\right.$ t.ha $\left.^{-1}\right)$ de acordo com a FAO (2013). Todos estes genótipos sobressaíram em relação aos melhores genótipos avaliados por Cavalcante et al. (2009) e Moreira et al. (2011), que encontraram 12,08 e 11,16 $\mathrm{t} \mathrm{ha} \mathrm{ha}^{-1}$, respectivamente. Tudo isso é indicativo da superioridade genética destes acessos. Embora a seleção de genótipos seja feita baseando-se geralmente na produtividade comercial de raízes, a produtividade total de raízes também é um parâmetro importante, pois as raízes não comercializáveis podem ser aproveitadas para a alimentação animal e para a produção de etanol (AZEVEDO et al., 2014).

Para a produtividade de matéria seca das raízes se destacaram os genótipos Cariru Vermelha, Licuri, Tomba Carro 1 e UFVJM (01, 21, 23, 28 e 40), com média de $13,0 \mathrm{t} \mathrm{ha}^{-1}$, valor $62 \%$ maior que o encontrado nas cultivares comerciais Brazlândia Branca e Brazlândia Rosada (Tabela 2). Todos estes genótipos também estão entre os de maiores produtividades de matéria fresca de raízes totais e comerciais, conforme o esperado. A produtividade de matéria seca é importante quando as raízes de batatadoce são destinadas à indústria alimentícia.

Quanto à produtividade de matéria fresca das ramas, destacaram-se os acessos Brazlandia Branca, Brazlandia Rosada, Cambraia, Cariru Vermelha, Espanhola, Palmas, Tomba Carro 1, UFVJM (01, 04, $05,07,14,18,21,23,25,28,38,39,40,41,44,46$ e 
56) com produtividade média de $37,47 \mathrm{t} \mathrm{ha}^{-1}$. Já os genótipos UFVJM-25, UFVJM-28 e UFVJM-41 se destacaram quanto à produtividade de matéria seca de ramas, com média de 12,67 t ha-1. Este valor é maior que o encontrado por Cavalcante et al. (2009), Cavalcante et al. (2010) e Figueiredo et al. (2012) em seus genótipos mais produtivos. Esta diferença pode ser explicada tanto pelos efeitos ambientais quanto genéticos (AZEVEDO et al., 2015). De forma geral, os acessos Tomba Carro 1, UFVJM 01 e UFVJM 28 podem ser considerados os mais produtivos devido à maior produtividade de matéria seca total, com média de 24,8 t ha ${ }^{-1}$. Além da alta produtividade de raízes, genótipos com alta produtividade de ramas devem ser preferidos, já que estas podem ser utilizadas para a alimentação animal tanto na forma in natura como na forma de silagem (VIANA et al., 2011).

Tabela 1. Análise descritiva e correlação de Pearson entre a produtividade (t ha $\left.{ }^{-1}\right)$ de características ${ }^{1}$ avaliadas em acessos de batata-doce do banco de germoplasma da UFVJM. Diamantina-MG, UFVJM. 2016.

\begin{tabular}{|c|c|c|c|c|c|c|c|c|c|c|}
\hline \multirow{2}{*}{ Parâmetros } & \multicolumn{10}{|c|}{ Análise Descritiva } \\
\hline & PTR & PRC & PMSR & PMFR & PMSRA & PMST & PAR & PPBR & PPBRA & PPBT \\
\hline Máximo $\left(\mathrm{t} \mathrm{ha}^{-1}\right)$ & 47,10 & 36,70 & 15,30 & 59,30 & 14,70 & 25,60 & 4,08 & 0,91 & 1,54 & 2,39 \\
\hline Media $\left(\mathrm{t} \mathrm{ha}^{-1}\right)$ & 28,71 & 18,52 & 8,93 & 28,81 & 5,85 & 14,78 & 1,35 & 0,53 & 0,74 & 1,27 \\
\hline Mínimo $\left(\mathrm{t} \mathrm{ha}^{-1}\right)$ & 16,70 & 9,10 & 4,70 & 3,59 & 0,70 & 6,40 & 0,00 & 0,30 & 0,10 & 0,59 \\
\hline $\begin{array}{l}\text { Desvio padrão } \\
\left(\mathrm{t} \mathrm{ha}^{-1}\right)\end{array}$ & 8,34 & 6,99 & 2,62 & 13,81 & 3,29 & 4,73 & 0,84 & 0,18 & 0,32 & 0,38 \\
\hline & 15,40 & 16,20 & 16,50 & 29,10 & 18,10 & 11,10 & 51,93 & 14,7 & 24,3 & 19,0 \\
\hline \multirow{2}{*}{ Características ${ }^{1}$} & \multicolumn{10}{|c|}{ Correlação de Pearson } \\
\hline & PTR & PRC & PMSR & PMFR & PMSRA & PMST & PAR & PPBR & PPBRA & PPBT \\
\hline PTR & 1,00 & $0,92^{* *}$ & $0,91^{* *}$ & $0,35^{*}$ & $0,28^{\mathrm{ns}}$ & $0,70^{* *}$ & $0,27^{\mathrm{ns}}$ & $0,82^{* *}$ & $0,35^{*}$ & $0,67^{* *}$ \\
\hline PRC & $0,92^{* *}$ & 1,00 & $0,77^{* *}$ & $0,30^{\mathrm{ns}}$ & $0,25^{\mathrm{ns}}$ & $0,60^{* *}$ & $0,30^{\text {ns }}$ & $0,70^{* *}$ & $0,34^{*}$ & $0,61^{* *}$ \\
\hline PMSR & $0,91^{* *}$ & $0,77^{* *}$ & 1,00 & $0,34^{*}$ & $0,27^{\mathrm{ns}}$ & $0,74^{* *}$ & $0,25^{\text {ns }}$ & $0,89^{* *}$ & $0,28^{\mathrm{ns}}$ & $0,65^{* *}$ \\
\hline PMFR & $0,35^{*}$ & $0,30^{\mathrm{ns}}$ & $0,34^{*}$ & 1,00 & $0,89^{* *}$ & $0,81^{* *}$ & $0,09^{\mathrm{ns}}$ & $0,15^{\mathrm{ns}}$ & $0,75^{* *}$ & $0,70^{* *}$ \\
\hline PMSRA & $0,28^{\text {ns }}$ & $0,25^{\mathrm{ns}}$ & $0,27 \mathrm{~ns}$ & $0,89^{* *}$ & 1,00 & $0,85^{* *}$ & $0,06^{\text {ns }}$ & $0,08^{\mathrm{ns}}$ & $0,80^{* *}$ & $0,71^{* *}$ \\
\hline PMST & $0,70^{* *}$ & $0,60^{* *}$ & $0,74^{* *}$ & $0,81^{* *}$ & $0,85^{* *}$ & 1,00 & $0,18^{\text {ns }}$ & $0,55^{* *}$ & $0,71^{* *}$ & $0,85^{* *}$ \\
\hline PAR & $0,27^{\mathrm{ns}}$ & $0,30^{\mathrm{ns}}$ & $0,25^{\mathrm{ns}}$ & $0,09^{\mathrm{ns}}$ & $0,06^{\mathrm{ns}}$ & $0,18^{\text {ns }}$ & 1,00 & $0,25^{\mathrm{ns}}$ & $0,03^{\text {ns }}$ & $0,14^{\mathrm{ns}}$ \\
\hline PPBR & $0,82^{* *}$ & $0,70^{* *}$ & $0,89^{* *}$ & $0,15^{\mathrm{ns}}$ & $0,08^{\mathrm{ns}}$ & $0,55^{* *}$ & $0,25^{\text {ns }}$ & 1,00 & $0,11^{\mathrm{ns}}$ & $0,56^{* *}$ \\
\hline PPBRA & $0,35^{*}$ & $0,34^{*}$ & $0,28^{n s}$ & $0,75^{* *}$ & $0,80^{* *}$ & $0,71^{* *}$ & $0,03^{\text {ns }}$ & $0,11^{\mathrm{ns}}$ & 1,00 & $0,89^{* *}$ \\
\hline BT & $0,67^{* *}$ & $0,61^{* *}$ & $0,65^{* *}$ & $0,70^{* *}$ & $0,71^{* *}$ & $0,85^{* *}$ & 0,14 & $0,56^{* *}$ & $0,89^{* *}$ & 1,00 \\
\hline
\end{tabular}

${ }^{1}$ Características: Produtividade total de raízes (PTR), produtividade de raízes comerciais (PRC), produtividade de matéria seca de raízes (PMSR), produtividade de matéria fresca das ramas (PMFR), produtividade de matéria seca de ramas (PMSRA), produtividade de matéria seca total (PMST), produtividade de amido nas raízes (PAR), produtividade de proteína bruta nas raízes (PPBR), produtividade de proteína bruta nas ramas (PPBRA) e produtividade de proteína bruta total (PPBT).

Para o acúmulo de amido, foi observado que os acessos Licuri, Palmas e UFVJM (01, 04, 14, 20 e 53) apresentaram produtividades maiores que $2,0 \mathrm{t} \mathrm{ha}^{-1}$, com destaque para Palmas e UFVJM-01, que produziram 3,53 e $4,08 \mathrm{t} \mathrm{ha}{ }^{-1}$, respectivamente, valores próximos aos produzidos em mandioca e milho (VILPOUX, 2008). Além do amido ser um dos principais carboidratos consumidos pelo homem (GONCCALVES et al., 2009), seu teor em batata-doce esta associado à produtividade de etanol (LÁZARI et al., 2014). Após avaliarem acessos superiores de batata-doce quanto à produtividade de etanol, Lázari et al. (2014) verificaram que produtividades de raízes equivalentes a $65,0 \mathrm{t} \mathrm{ha}^{-1}$, com concentração de amido nas raízes próxima a $25 \%$, conferem rendimento de etanol superior a $10.000 \mathrm{~L} \mathrm{ha}^{-1}$. Segundo Gonçalves Neto et al. (2011) alguns genótipos de batata-doce apresentam superioridade na produção de etanol quando comparados à cana-de-açúcar. Logo, os acessos Palmas e UFVJM (01, $04,05,07$ e 53) são os mais promissores quanto à produtividade de etanol.

Segundo Apata \& Babalola (2012) as plantas de batata-doce possuem boas qualidades nutricionais, sendo superiores a outras fontes utilizadas para a alimentação animal, e consequentemente, tem potencial de utilização na alimentação de animais ruminantes e não-ruminantes. Para a atividade normal das bactérias ruminais são necessários 
Tabela 2. Produtividade $\left(\mathrm{t} \mathrm{ha} \mathrm{a}^{-1}\right)$ de raízes totais (PTR), de raízes comerciais (PRC), de matéria seca de raízes (PMSR), de matéria fresca das ramas (PMFR), de matéria seca de ramas (PMSRA), de matéria seca total (PMST), de amido nas raízes (PAR), de proteína bruta nas raízes (PPBR), de proteína bruta nas ramas (PPBRA) e de proteína bruta total (PPBT) em acessos de batata-doce do banco de germoplasma da UFVJM. Diamantina-MG, UFVJM. 2016.

\begin{tabular}{|c|c|c|c|c|c|c|c|c|c|c|}
\hline Genótipo & PTR & PRC & PMSR & PMFR & PMSRA & PMST & PAR & PPBR & PPBRA & PPBT \\
\hline Bat. Mandioca & $43,5 \mathrm{a}$ & $36,7 \mathrm{a}$ & 9,4 b & $5,86 \mathrm{~b}$ & $1,1 \mathrm{c}$ & $10,5 \mathrm{c}$ & 1,88 & $0,62 \mathrm{~b}$ & $0,79 \mathrm{~b}$ & $1,41 \mathrm{~b}$ \\
\hline Braz. Branca & $25,4 \mathrm{~b}$ & $18,8 \mathrm{~b}$ & $7,4 \mathrm{~b}$ & $37,6 \mathrm{a}$ & $7,3 \mathrm{~b}$ & $14,7 \mathrm{~b}$ & 1,55 & $0,36 \mathrm{c}$ & $84 \mathrm{a}$ & $20 \mathrm{~b}$ \\
\hline Braz.Rosada & $30,2 \mathrm{~b}$ & $24,2 \mathrm{a}$ & $8,7 \mathrm{~b}$ & $43,5 \mathrm{a}$ & $9,4 \mathrm{~b}$ & $1 \mathrm{~b}$ & 1,74 & $0,48 \mathrm{~b}$ & $1,11 \mathrm{a}$ & $1,59 \mathrm{~b}$ \\
\hline Cambraia & $30,7 \mathrm{~b}$ & $19,7 \mathrm{~b}$ & $10,2 \mathrm{~b}$ & $32,3 \mathrm{a}$ & $8,6 \mathrm{~b}$ & $18,8 \mathrm{~b}$ & 1,63 & $0,64 \mathrm{~b}$ & $0,79 \mathrm{~b}$ & $1,43 \mathrm{~b}$ \\
\hline C. Vermelha & $42,3 \mathrm{a}$ & $29,5 \mathrm{a}$ & $14,9 \mathrm{a}$ & $31,8 \mathrm{a}$ & $6,4 \mathrm{~b}$ & $21,3 \mathrm{~b}$ & 0,00 & $0,88 \mathrm{a}$ & $90 \mathrm{a}$ & $1,78 \mathrm{~b}$ \\
\hline Espanhola & $25,9 \mathrm{~b}$ & $16,5 \mathrm{~b}$ & $8,3 \mathrm{~b}$ & $29,3 \mathrm{a}$ & $4,0 \mathrm{c}$ & $12,3 \mathrm{~b}$ & 1,81 & $0,52 \mathrm{~b}$ & $0,60 \mathrm{~b}$ & $1,12 \mathrm{c}$ \\
\hline Licuri & $35,9 \mathrm{a}$ & $27,2 \mathrm{a}$ & $12,3 \mathrm{a}$ & $19,8 \mathrm{~b}$ & $3,2 \mathrm{c}$ & $5 \mathrm{~b}$ & 2,17 & $0,81 \mathrm{a}$ & $0,41 \mathrm{~b}$ & $1,22 \mathrm{~b}$ \\
\hline Palmas & $28,9 \mathrm{~b}$ & $19,4 \mathrm{~b}$ & $10,1 \mathrm{~b}$ & $29,3 \mathrm{a}$ & $4,9 \mathrm{c}$ & $15,0 \mathrm{~b}$ & 3,53 & $0,53 \mathrm{~b}$ & $0,58 \mathrm{~b}$ & $1,11 \mathrm{c}$ \\
\hline T. Carro 1 & $47,1 \mathrm{a}$ & $25,8 \mathrm{a}$ & $15,3 \mathrm{a}$ & $59,3 \mathrm{a}$ & $10,3 \mathrm{~b}$ & $6 \mathrm{a}$ & 0,89 & $0,87 \mathrm{a}$ & $1,09 \mathrm{a}$ & $1,96 \mathrm{a}$ \\
\hline UFVJM-01 & $41,0 \mathrm{a}$ & $28,5 \mathrm{a}$ & $13,2 \mathrm{a}$ & $53,7 \mathrm{a}$ & $11,8 \mathrm{~b}$ & $0 \mathrm{a}$ & 4,08 & $0,91 \mathrm{a}$ & $1,48 \mathrm{a}$ & $2,39 \mathrm{a}$ \\
\hline UF & $18,1 \mathrm{c}$ & $9,8 \mathrm{c}$ & $6,6 \mathrm{c}$ & $23,7 \mathrm{~b}$ & 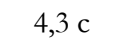 & $9 \mathrm{c}$ & 0,76 & $0,34 \mathrm{c}$ & $0,59 \mathrm{~b}$ & $0,93 \mathrm{c}$ \\
\hline UFVJM-04 & $28,4 \mathrm{~b}$ & $16,4 \mathrm{~b}$ & $9,1 \mathrm{~b}$ & $34,5 \mathrm{a}$ & 6,2 & 15 & 2,28 & $0,44 \mathrm{c}$ & $0,87 \mathrm{a}$ & $1,30 \mathrm{~b}$ \\
\hline UFVJM-05 & $17,6 \mathrm{c}$ & $9,4 \mathrm{c}$ & $6,1 \mathrm{c}$ & $35,0 \mathrm{a}$ & 7 , & & 1,55 & $0,30 \mathrm{c}$ & $0 \mathrm{a}$ & $1,20 \mathrm{~b}$ \\
\hline M-06 & $38,0 \mathrm{a}$ & $23,1 \mathrm{a}$ & $9,8 \mathrm{~b}$ & $23,2 \mathrm{~b}$ & 4,9 & 14 & 1,51 & $0,61 \mathrm{~b}$ & $0,63 \mathrm{~b}$ & $1,24 \mathrm{~b}$ \\
\hline UF & $18,4 \mathrm{c}$ & $9,1 \mathrm{c}$ & $6,0 \mathrm{c}$ & $28,8 \mathrm{a}$ & $5,4 \mathrm{c}$ & c & 1,50 & $8 \mathrm{c}$ & $8 \mathrm{~b}$ & $1,06 \mathrm{c}$ \\
\hline UFVJM-12 & $26,3 \mathrm{~b}$ & $19,0 \mathrm{~b}$ & $8,0 \mathrm{~b}$ & $26,1 \mathrm{~b}$ & $3,5 \mathrm{c}$ & $11,5 \mathrm{c}$ & 0,51 & $0,41 \mathrm{c}$ & $0,40 \mathrm{~b}$ & $0,81 \mathrm{c}$ \\
\hline M-14 & $29,1 \mathrm{~b}$ & $20,1 \mathrm{~b}$ & $9,8 \mathrm{~b}$ & $31,3 \mathrm{a}$ & $5,4 \mathrm{c}$ & $15,2 \mathrm{~b}$ & 2,16 & $0,48 \mathrm{~b}$ & $0,73 \mathrm{~b}$ & $1,21 \mathrm{~b}$ \\
\hline UFV & $25,3 \mathrm{~b}$ & $16,2 \mathrm{~b}$ & $9,4 \mathrm{~b}$ & $31,0 \mathrm{a}$ & 8,5 & 17 & 0,71 & $0,48 \mathrm{~b}$ & $0,88 \mathrm{a}$ & $1,36 \mathrm{~b}$ \\
\hline UFVJM-20 & $32,2 \mathrm{~b}$ & $16,3 \mathrm{~b}$ & $10,6 \mathrm{~b}$ & $22,6 \mathrm{~b}$ & $4,1 \mathrm{c}$ & $14,7 \mathrm{~b}$ & 2,14 & $0,63 \mathrm{~b}$ & $0,50 \mathrm{~b}$ & $1,13 \mathrm{c}$ \\
\hline UFVJM-21 & $44,0 \mathrm{a}$ & $27,1 \mathrm{a}$ & $14,1 \mathrm{a}$ & $46,4 \mathrm{a}$ & $6,6 \mathrm{c}$ & 20 & 0,90 & $0,90 \mathrm{a}$ & $0,71 \mathrm{~b}$ & $1,62 \mathrm{~b}$ \\
\hline UFVJM-23 & $38,9 \mathrm{a}$ & $27,6 \mathrm{a}$ & $12,3 \mathrm{a}$ & $27,4 \mathrm{a}$ & 6,9 & $19,2 \mathrm{~b}$ & 1,91 & $0,81 \mathrm{a}$ & $0,72 \mathrm{~b}$ & $1,53 \mathrm{~b}$ \\
\hline UFVJM-24 & $30,2 \mathrm{~b}$ & $20,5 \mathrm{~b}$ & $8,4 \mathrm{~b}$ & $22,2 \mathrm{~b}$ & $4,2 \mathrm{c}$ & $12,6 \mathrm{~b}$ & 1,64 & $0,50 \mathrm{~b}$ & $0,53 \mathrm{~b}$ & $1,04 \mathrm{c}$ \\
\hline UFVJM-25 & $25,6 \mathrm{~b}$ & $15,2 \mathrm{~b}$ & $8,4 \mathrm{~b}$ & $38,8 \mathrm{a}$ & $11,5 \mathrm{a}$ & $19,9 \mathrm{~b}$ & 1,85 & $0,49 \mathrm{~b}$ & $0,66 \mathrm{~b}$ & $1,15 \mathrm{c}$ \\
\hline UFVJM-28 & $43,1 \mathrm{a}$ & $31,6 \mathrm{a}$ & $12,1 \mathrm{a}$ & $48,5 \mathrm{a}$ & $11,8 \mathrm{a}$ & 23 & 0,89 & $0,62 \mathrm{~b}$ & $1,39 \mathrm{a}$ & $2,01 \mathrm{a}$ \\
\hline UFVJM-38 & $24,0 \mathrm{~b}$ & $16,1 \mathrm{~b}$ & $6,8 \mathrm{c}$ & $28,3 \mathrm{a}$ & $5,6 \mathrm{c}$ & $12,4 \mathrm{~b}$ & 0,75 & $0,30 \mathrm{c}$ & $0,71 \mathrm{~b}$ & $1,00 \mathrm{c}$ \\
\hline UFVJM & $20,1 \mathrm{c}$ & $10,1 \mathrm{c}$ & $6,2 \mathrm{c}$ & $38,0 \mathrm{a}$ & $9,2 \mathrm{~b}$ & $15,4 \mathrm{~b}$ & 0,49 & $0,35 \mathrm{c}$ & $1,19 \mathrm{a}$ & $1,53 \mathrm{~b}$ \\
\hline UFVJM-40 & $32,9 \mathrm{a}$ & $22,7 \mathrm{a}$ & $10,9 \mathrm{a}$ & $35,3 \mathrm{a}$ & $6,1 \mathrm{~b}$ & $17,0 \mathrm{~b}$ & 1,19 & $0,61 \mathrm{~b}$ & $0,84 \mathrm{a}$ & $1,45 \mathrm{~b}$ \\
\hline UFVJM-41 & $26,5 \mathrm{~b}$ & $18,3 \mathrm{~b}$ & $6,4 \mathrm{c}$ & $56,2 \mathrm{a}$ & $14,7 \mathrm{a}$ & $21,1 \mathrm{~b}$ & 0,55 & $0,38 \mathrm{c}$ & $1,54 \mathrm{a}$ & $1,93 \mathrm{a}$ \\
\hline UFVJM-44 & $21,0 \mathrm{c}$ & $11,9 \mathrm{c}$ & $5,9 \mathrm{c}$ & $33,5 \mathrm{a}$ & & $12,4 \mathrm{~b}$ & 0,54 & $0,38 \mathrm{c}$ & $0,69 \mathrm{~b}$ & $1,07 \mathrm{c}$ \\
\hline UFVJM-46 & $21,0 \mathrm{c}$ & $12,0 \mathrm{c}$ & $6,2 \mathrm{c}$ & $41,7 \mathrm{a}$ & $7,2 \mathrm{~b}$ & $13,4 \mathrm{~b}$ & 0,45 & $0,31 \mathrm{c}$ & $0,84 \mathrm{a}$ & $1,15 \mathrm{c}$ \\
\hline UFVJM-47 & $29,6 \mathrm{~b}$ & $16,4 \mathrm{~b}$ & $8,7 \mathrm{~b}$ & $20,8 \mathrm{~b}$ & $5,4 \mathrm{c}$ & $14,1 \mathrm{~b}$ & 0,96 & $0,43 \mathrm{c}$ & $0,61 \mathrm{~b}$ & $1,04 \mathrm{c}$ \\
\hline UFVJM-48 & $23,1 \mathrm{~b}$ & $16,6 \mathrm{~b}$ & $7,9 \mathrm{~b}$ & $18,0 \mathrm{~b}$ & $4,4 \mathrm{c}$ & $12,3 \mathrm{c}$ & 0,62 & $0,49 \mathrm{~b}$ & $0,59 \mathrm{~b}$ & $1,08 \mathrm{c}$ \\
\hline UFVJM-50 & $19,1 \mathrm{c}$ & $11,1 \mathrm{c}$ & $7,0 \mathrm{~b}$ & $11,7 \mathrm{~b}$ & $3,8 \mathrm{c}$ & $10,8 \mathrm{c}$ & 0,67 & $0,41 \mathrm{c}$ & $0,47 \mathrm{~b}$ & $0,88 \mathrm{c}$ \\
\hline UFVJM-51 & $22,6 \mathrm{~b}$ & $11,2 \mathrm{c}$ & $7,9 \mathrm{~b}$ & $5,3 \mathrm{~b}$ & $2,3 \mathrm{c}$ & $10,2 \mathrm{c}$ & 0,68 & $0,54 \mathrm{~b}$ & $0,33 \mathrm{~b}$ & $0,87 \mathrm{c}$ \\
\hline UFVJM-52 & $18,8 \mathrm{c}$ & $11,4 \mathrm{c}$ & $6,1 \mathrm{c}$ & $5,11 \mathrm{~b}$ & $1,9 \mathrm{c}$ & $8,0 \mathrm{c}$ & 1,31 & $0,37 \mathrm{c}$ & $0,25 \mathrm{~b}$ & $0,62 \mathrm{c}$ \\
\hline UFVJM-53 & $22,5 \mathrm{~b}$ & $11,9 \mathrm{c}$ & $6,5 \mathrm{c}$ & $13,3 \mathrm{~b}$ & $1,2 \mathrm{c}$ & $7,7 \mathrm{c}$ & 2,06 & $0,42 \mathrm{c}$ & $0,17 \mathrm{~b}$ & $0,59 \mathrm{c}$ \\
\hline UFVJM-55 & $16,7 \mathrm{c}$ & $10,6 \mathrm{c}$ & $4,7 \mathrm{c}$ & $22,2 \mathrm{~b}$ & $1,7 \mathrm{c}$ & $6,4 \mathrm{c}$ & 0,59 & $0,39 \mathrm{c}$ & $0,73 \mathrm{~b}$ & $1,12 \mathrm{c}$ \\
\hline UFVJM-56 & $33,4 \mathrm{a}$ & $25,5 \mathrm{a}$ & $8,5 \mathrm{~b}$ & $27,7 \mathrm{a}$ & $5,2 \mathrm{c}$ & $13,7 \mathrm{c}$ & 1,99 & $0,48 \mathrm{~b}$ & $0,72 \mathrm{~b}$ & $1,20 \mathrm{~b}$ \\
\hline & $27,5 \mathrm{~b}$ & $11,6 \mathrm{c}$ & $9,7 \mathrm{~b}$ & $3,59 \mathrm{~b}$ & $0,9 \mathrm{c}$ & $10,6 \mathrm{c}$ & 0,81 & $0,62 \mathrm{~b}$ & $0,95 \mathrm{a}$ & $1,57 \mathrm{~b}$ \\
\hline UFVJM-58 & $23,7 \mathrm{~b}$ & $15,7 \mathrm{~b}$ & $7,1 \mathrm{~b}$ & $9,66 \mathrm{~b}$ & $0,7 \mathrm{c}$ & $7,7 \mathrm{c}$ & 0,82 & $0,75 \mathrm{a}$ & $0,10 \mathrm{~b}$ & $0,84 \mathrm{c}$ \\
\hline
\end{tabular}

Médias seguidas por mesma letra, na coluna, não diferem entre si pelo agrupamento de Scott-Knott em nível de 5\% de probabilidade. 
de 6 a $8 \%$ de proteína bruta (FIGUEIREDO et al., 2012). Nos genótipos avaliados neste trabalho, os teores de proteína bruta variaram entre 10 a $14 \%$ na parte aérea, e entre 5 a 10,5\% nas raízes. Desta forma, plantas de batata-doce podem ser indicadas para substituição ou adição a outros alimentos comumente utilizados como rações para animais, como girassol, farelo de arroz e sorgo (REZENDE et al., 2002). Além da proteína bruta, a cultura possui outros atributos nutricionais adequados, como teor de fibras (APATA \& BABALOLA 2012; ANDRADE JÚNIOR et al. 2012), aminoácidos, pH (MONTEIRO et al., 2007; VIANA et al., 2011), elementos minerais (RÉGNIER et al., 2012), dentre outros. Assim, as produtividades médias de proteína bruta obtidas nesse trabalho variando de 0,59 a $2,39 \mathrm{t} \mathrm{ha}^{-1}$, mostrando a variabilidade entre os materiais e a possibilidade de seleção de acessos de interesse agropecuário. Os acessos Cariru Vermelha, Licuri, Tomba Carro1 e UFVJM (01, 21, 23 e 58) produziram, em valores médios, $0,85 \mathrm{t} \mathrm{ha}^{-1}$ de proteína bruta nas raízes. Já para a parte aérea, os acessos Brazlândia Branca, Brazlândia Rosada, Cariru Vermelha, Tomba Carro1 e UFVJM $(01,04,05,18,28,39,40,41,46$ e 57$)$ se destacaram, com produtividade média de $1,0 \mathrm{t} \mathrm{ha}^{-1}$. Por fim, os acessos Tomba Carro 1 e UFVJM (01, 28 e 41) foram superiores no acúmulo total (ramas + raízes) de proteína bruta com produtividade média de 2,07 t ha-1 (Tabela 2).

O potencial produtivo da batata-doce é inquestionável. A produtividade de matéria seca total (raízes + ramas) obtida para o acesso Tomba Carro 1 (25,60 $\left.\mathrm{t} \mathrm{ha}^{-1}\right)$ supera muito as encontradas para capimbraquiária $\left(14,05 \mathrm{t} \mathrm{ha}^{-1}\right)$, milho $\left(21,10 \mathrm{t} \mathrm{ha}^{-1}\right)$, sorgosudão $\left(19,61 \mathrm{t} \mathrm{ha}^{-1}\right)$, sorgo forrageiro $\left(23.15 \mathrm{t} \mathrm{ha}^{-1}\right) \mathrm{e}$ girassol (15,92 $\left.\mathrm{t} \mathrm{ha}^{-1}\right)$ (Leonel et al., 2008, Oliveira et al., 2010). Da mesma forma, a produtividade de proteína bruta obtida em toda a planta (raízes + ramas) no acesso UFVJM-01 (2,39 $\left.\mathrm{t} \mathrm{ha}^{-1}\right)$ supera bastante a encontrada em amendoim forrageiro $\left(0,69 \mathrm{t} \mathrm{ha}^{-1}\right)$, milho $\left(0,68 \mathrm{t} \mathrm{ha}^{-1}\right)$, capim elefante $\left(0,50 \mathrm{t} \mathrm{ha}^{-1}\right)$, capimbraquiária $\left(0,58 \mathrm{t} \mathrm{ha}^{-1}\right)$, sorgo $\left(0,80 \mathrm{t} \mathrm{ha}^{-1}\right)$ e estilozante $\left(0,25 \mathrm{t} \mathrm{ha}^{-1}\right)$ (Miranda et al. 2003, Leonel et al. 2008, Moura et al. 2011). Logo, o potencial produtivo da batata-doce aliado à sua rusticidade e múltiplos fins, torna a cultura de alta importância e relevância. Consequentemente, esforços devem ser conduzidos no estudo da cultura e no estímulo de seu cultivo.

\section{CONCLUSÕES}

Os acessos Tomba Carro 1, UFVJM-01, UFVJM-28 e UFVJM-40 possuem maior potencial produtivo de raízes, ramas e proteína bruta, sendo adequados à alimentação humana e animal. Os acessos Palmas e UFVJM (01, 04, 05, 07 e 53) podem ter maior potencial para a produção de etanol devido à maior produtividade de amido nas raízes. Quanto à produção na planta (raízes e rama), destacaram-se os acessos T. Carro 1, UFVJM-01 e UFVJM-28, com maiores produtividades de matéria seca total e proteína bruta total.

\section{AGRADECIMENTOS}

Ao CNPq, FAPEMIG e CAPES pela concessão de bolsas de estudos e recursos financeiros para o desenvolvimento do projeto.

\section{REFERÊNCIAS}

ANDRADE JÚNIOR, V. C.; VIANA, D. J. S.; FERNANDES, J. S. C.; FIGUEIREDO, J. A.; NUNES, U. R.; NEIVA, I. P. Selection of sweet potato clones for the region Alto Vale do Jequitinhonha. Horticultura Brasileira, v. 27, n. 3, p. 389393, set. 2009.

APATA, D. F.; BABALOLA, T. O. The use of cassava, sweet potato and cocoyam, and their byproducts by non ruminants. International Journal of Food Science and Nutrition Engineering, v. 2, n. 4, p. 54-62, 2012.

AZEVEDO, A. M.; ANDRADE JÚNIOR, V. C.; FERNANDES, J. S. C.; PEDROSA, C. E.; OLIVEIRA, C. M. Desempenho agronômico e parâmetros genéticos em genótipos de batata-doce. Horticultura Brasileira, v. 33, n. 1, p. 84-90, 2015.

AZEVEDO, A. M.; ANDRADE JÚNIOR, V. C.; VIANA, D. J. S.; ELSAYED, A. Y. A. M.; PEDROSA, C. E.; NEIVA, I.; FIGUEIREDO, A. F. Influence of harvest time and cultivation sites on the productivity and quality of sweet potato. Horticultura Brasileira, v. 32, n. 1, p. 21-27, 2014.

CARDOSO, A. D.; VIANA, A. E. S.; MATSUMOTO, S. N.; BONFIM NETO, H.; KHOURI, C. R.; MELO, T. L. Características físicas e sensoriais de clones de batata-doce. Ciência e Agrotecnologia, v. 31, n. 6, p. $1760-$ 1765, 2007. 
CAVALCANTE, M.; FERREIRA, P. V.; PAIXÃO, S. L.; COSTA, J. G.; PEREIRA, R. G.; MADALENA, J. M. S. Desempenho agronômico, dissimilaridade genética e seleção de genitores de batata-doce para hibridização. Revista Brasileira de Ciências Agrárias, v. 5, n. 4, p. 485-490, 2010.

CAVALCANTE, M; FERREIRA, P. V.; PAIXÃO, S. L.; COSTA, J. C.; PEREIRA, R. G.; MADALENA, J. A. S. Potenciais produtivo e genético de clones de batata-doce. Acta Scientiarum. Agronomy, v. 31, n. 3, p. 421-426, 2009.

CORDENUNSI, B. R.; GENOVESE, M. I.; NASCIMENTO, J. R. O.; HASSIMOTTO, N. M. A.; SANTOS, N. M. A.; LAJOLO, F. M. Effects on the temperature on the chemical composition and antioxidant activity of three strawberry cultivars. Food Chemistry, v. 91, p. 113-121, 2005.

FAO - Food and agriculture organization of the united nations. Sistema de dados agricolas atualizados. Disponível em: < http://faostat.fao.org/site> Acesso em: 22 abril 2013.

FIGUEIREDO, J. A.; ANDRADE JÚNIOR, V. C.; PEREIRA, R. C.; RIBEIRO, K. G.; VIANA, D. J. S.; NEIVA, I. P. Avaliação de silagens de ramas de batatadoce. Horticultura Brasileira, v. 30, n. 4, p. 708-712, 2012.

GONÇALVES NETO, A. C.; MALUF, W. R.; GOMES, L. A. A.; GONÇALVES, R. J. S.; SILVA, V. F.; LASMAR, A. Aptidões de genótipos de batata-doce para consumo humano, produção de etanol e alimentação animal. Pesquisa Agropecuária Brasileira, v. 46, n. 11, p. 1513-1520, 2011.

GONÇALVES NETO, A. C.; MALUF, W. R.; GOMES, L. A. A.; MACIEL, G. M.; FERREIRA, R. P. D.; CARVALHO, R. C. Correlação entre caracteres e estimação de parâmetros populacionais para batatadoce. Horticultura Brasileira, v. 30, n. 4, p. 713-719, 2012.

GONÇALVES, M. F. V.; SARMENTO, S. B. S.; DIAS, C. T. S.; MARQUEZINI, N. Tratamento térmico do amido de batata-doce (Ipomoea batatas L.) sob baixa umidade em micro-ondas. Ciência e Tecnologia de Alimentos, v. 29, n. 2, p. 270-276, 2009.
LÁZARI, T. M.; SILVEIRA, M. A.; DOURADO, D. P.; MIRANDA, F. F. R; MURAISHI, C. T. Path analysis in characteristics of sweet potato clones aiming ethanol yield. Brazilian Journal of Applied Technology for Agricultural Science, v. 7, n. 2, p. 41-47, 2014

LEONEL, F. P.; PEREIRA, J. C.; COSTA, M. G.; MARCO JÚNIOR, P.; LARA, L. A.; RIBEIRO, M. D.; SILVA, C. J. Consórcio capim-braquiária e milho: produtividade das culturas e características qualitativas das silagens feitas com plantas em diferentes idades. Revista Brasileira de Zootecnia, v. 37, n. 12, p. 2233-2242, 2008.

MARCHESE, A.; MALUF, W. R.; GONÇALVES NETO, A. C.; GONÇALVES, R. D. S.; GOMES, L. A. A. Seleção de clones de batata-doce resistentes a Meloidogyne incognita raça 1. Pesquisa Agropecuária Brasileira, v. 45 , n. 9, p. 997-1004, 2010.

MCCREADY, R. M.; GUGGOLZ, J.; SILVIERA, V.; OWENS, H. S. Determination of starch and amylose in vegetables. Application to peas. Analytical Chemistry, v. 22, n. 9, p. 1156-1158, 1950.

MIRANDA, C. H. B.; VIEIRA, A.; CADISCH, G. Determinação da fixação biológica de nitrogênio no amendoim forrageiro (Arachis spp.) por intermédio da abundância natural de $15 \mathrm{~N}$. Revista Brasileira de Zootecnia, v. 32, n. 6, p. 1859-1865, 2003.

MONTEIRO, A. B. Silagens de cultivares e clones de batata-doce para alimentação animal visando sustentabilidade da produção agrícola familiar. Revista Brasileira de Agroecologia, v. 2, n. 2, p. 978-981, 2007.

MOREIRA, J. N.; QUEIROGA, R. C. F.; SOUSA JÚNIOR, A. J. L.; SANTOS, M. A. Caracteres morfofisiológicos e produtivos de cultivares de batatadoce, em Mossoró, RN. Revista Verde, v. 6, n. 11, p. 161-167, 2011.

MOURA, R. L.; NASCIMENTO, M. P. S. C. B.; RODRIGUES, M. M.; OLIVEIRA, M. E.; LOPES, J. B. Razão folha/haste e composição bromatológica da rebrota de estilosantes campo grande em cinco idades de corte. Acta Scientiarum. Animal Sciences, v. 33, n. 3, p. 249-254, 2011. 
OLIVEIRA, L. B.; PIRES, A. J. V.; VIANA, A. E. S.; MATSUMOTO, S. N.; CARVALHO, G. G. P.; RIBEIRO, L. S. O. Produtividade, composição química e características agronômicas de diferentes forrageiras. Revista Brasileira de Zootecnia, v. 39, n. 12, p. 2604-2610, 2010.

RÉGNIER, C.; JAGUELIN, Y.; NOBLET, J.; RENAUDEAU, D. Ileal digestibility of amino acids of cassava, sweet potato, cocoyam and erythrina foliages fed to growing pigs. Animal, v. 6, n. 4, p. 586-593, 2012.
REZENDE, A. V.; EVANGELISTA, A. R.; SIQUEIRA, G. R.; SANTOS, R. V.; SALES, E. C. J.; BERNARDES, T. F. Avaliação do potencial do girassol (Helianthus annuus L.) como planta forrageira para ensilagem na safrinha, em diferentes épocas de cortes. Ciência e Agrotecnologia, v. 35, n.5, p. 1548-1553, 2002.

VIANA, D. J. S.; ANDRADE JÚNIOR, V. C.; RIBEIRO, K. G.; PINTO, N. A. V. D.; NEIVA, I. P.; FIGUEIREDO, J. A.; LEMOS, V. T.; PEDROSA, C. E.; AZEVEDO, A. M. Potencial de silagens de ramas de batata-doce para alimentação animal. Ciência Rural, v. 41, n. 8, p. 1466-1471, 2011.

VILPOUX, O. F. Competitividade da mandioca no Brasil, como matéria-prima para amido. Informações Econômicas, v. 38, n. 11, p. 27-38, 2008. 